\title{
Integrated Wireless Technologies with Computer for Industrial Machinery Fault Diagnosis: Challenges Comparison and Characteristics: A Review
}

\author{
Moneer Ali Lilo, ${ }^{1, *}$, Auda Raheemah Odhaib ${ }^{2}$, Abdelkrim K. Ilijanb ${ }^{3}$ \\ ${ }^{1}$ Department of Physics, College of Science, Al Muthanna University, Al Muthanna, Iraq \\ ${ }^{2}$ College of Science, Al Muthanna University, Al Muthanna, Iraq \\ ${ }^{3}$ Engineering College, Al Muthanna University, Al Muthanna, Iraq \\ *Corresponding Author: moneerlilo@yahoo.com
}

Received 15/4/2018, Accepted 15/5/2018, published 2018.

DOI: 10.52113/2/05.01.2018/61-75

\begin{abstract}
Condition monitoring of machinery in industries is becoming an emerging technique for efficient operations and productivity. A wide range of fault diagnosis approaches have been proposed to improve machinery operations in industries. These monitoring and fault diagnosis approaches are most effective for reliable machinery operations. However, due to the harsh environment of industries, these approaches have been suffered from different challenges. In order to extract the significant features for fault diagnosis and monitoring, different neural, fuzzy, and signal processing based systems were adopted. In this paper, we discuss wireless sensor based fault diagnosis and monitoring approaches and their types for industrial machinery. Furthermore, the paper presents industrial challenges to adopting these approaches and related efforts.
\end{abstract}

C2018 Al Muthanna University. All rights reserved

Keywords: Fuzzy, Neural Network, Vibration Fault, Accuracy, Fault Diagnosis, Wireless system

\section{Introduction}

Recently, wireless communication technologies have been implemented in all fields of life and play a significant role to enhance the performance of different operations. The usage of these technologies in industrial area diverts all processes and other machinery operations into advanced and cost effective way. The industrial machinery processes are often composed with various devices and equipment. However, the traditional wired mechanism makes the industrial environment more critical and congested and always leads to maintenance and fault diagnosis issues. Industries always looking for effective and efficient maintenance procedures and solution for better productivity. The one of the novel technique is condition-based maintenance, which decides the maintenance time based on machinery condition and improves the machinery condition. Condition based applications are used to monitor and facilitate the early faults detection will protect the machine from damage caused by faults, and early protective actions will be taken. Wireless sensors technique overtakes many challenges existed in wired systems such as maintenance, cost, installation, and un-flexibility to change the sensor location. Thus, researchers are utilizing the WSNs, while WSNs are still require further enhancement in WSN such as for reducing real time processing, improving data transmission reliability, and the signal processing. 
With many advantages, WSNs have some limitations in terms limited radio range, energy and computational complexities. Several different types of WSN based solutions have been proposed to address these constraints. Most of WSN applications have been used for data acquisition and transmission and extract the fault diagnosis functions. These types of diagnosis are promising alternative approach to transmitting the raw data and reduce the quality and save energy and time. In many industrial WSN applications, it is difficult to obtain all information about the device and their faults by a single sensor node. Due to the harsh environment of industries, the wireless-based devices have been suffered in communication quality in terms of noise and interference. These issues disturb the communication quality and increase uncertainty in diagnosis results. Data fusion was presented to augment the accuracy of transmitting process based on combining different types of data into one packet. It is effective method to save energy and computation power such as fuzzy data fusion [1], Baysian method [2], Dempster-Shafer theory [3]. These methods are based on wired sensor systems and have some limitations.

The main aim of this paper is to discuss the fault diagnosis and machine monitoring approaches. We also discuss the wireless sensor networks in industrial applications and reviewed the network types, protocol standards, and recent challenges to adopting these technologies. Furthermore, the paper presents industrial wireless sensor networks and different sensors in industries. In addition, proposed fault diagnosis and monitoring approaches are discussed in related work section.

\section{WSN Overview}

The WSNs were developed for monitoring and controlling usages in industry, health care, and greenhouse. Thus, the complexity of data transmission and reception is reduced. Moreover, these applications enhance the efficiency and reduce the cost in industrial environment. The WSNs are existing in industrial applications for fault detection, machine or equipment control, data collection, monitoring system situation, and real-time communication [4]. The tiny sensors revolution with the property of wireless technique provided the ability to install these sensors on equipment of the expensive machines. These sensors are utilized for monitoring and protecting the machine from the damage caused by faults such as temperature, pressure, vibration, and flow if the fault is in the danger zone. Thus, sensors are collecting and transmitting the data to a central computer or sink node for analysis, processing, and generating action to protect the machine. Therefore, utilizing wireless sensors will reduce the cost and time of the maintenance when catastrophic failures happen in the industrial environment.

\subsection{Network Types}

The network topology is considered as a backbone of systems and also significant for industrial communications. The network topology designs on the basis of connectivity requirements, mobility, adaptability and scalability. There are various important factors take into account to adopt network topology in industries such as how the system adopt There are various important factors taken into account to adopt network topology in industries such as; how the system adopt, device, frequency, power consumption, latency, compatibility to add new sensors to the network. Mostly, there are three topology types, which are selected based on many parameters. These topologies are; mesh, star, and star mesh as illustrated in Figure (1). 


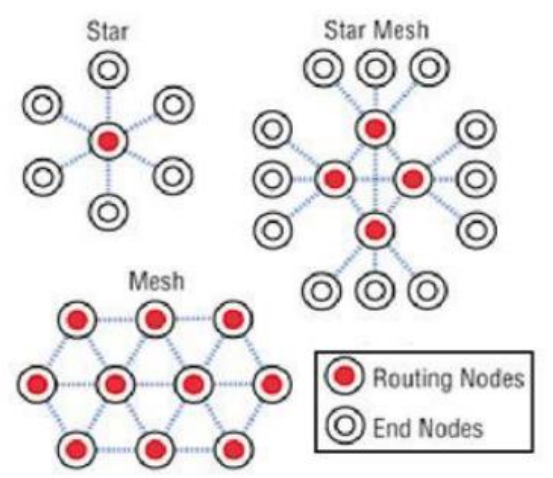

Fig.(1): IWSN Network topologies [4].

In start network, every node has a designated as a forwarding path. This type of strategy fails when two nodes are not communicating due to any fault and information is lost. To address this issue in start network, the star with mesh network was introduced. The start mesh network is scalable, adaptable and able to deal with mobility. However, this type of network does not support end-to-end redundancy. In mesh topology, each sensor is with full redundancy and fully connected with the network coordinator. The end node (sensor) will send the collected data to the sink node depending on multi-path algorithms. Therefore, if there is a path failure during data transmission, the data will be transmitted to the destination point via other paths. The main properties of mesh topology are: self-organizing, scalability, and high adaptively. This topology enhanced the WSN performance in industrial applications, due to the ability for easy expanding the via reducing the packet size. Basically, data fusion is a localized mechanism for data processing in IWSN for minimizing flooding and redundancy. The cross-layer designing is another significant need for handle the communication layers of protocols [5]. Various working groups have established network by adding some nodes to the main network.With diverse benefits, mesh topology has a disadvantage related to processing time, due to the data send, and processing from multi-path that increases the delay time processing.

\subsection{Prototypes}

In IWSN, efficient and scalable network architecture is necessary to fulfill the industrial requirements. The network has capabilities to deploy homogenous sensors and able to program every node with all possible applications tasks. This type of strategy produces a flat single-tier network for sensors. On the other hand, the heterogeneous elements utilized by the multitier approach. In multitier approach, the low power sensors performing simpler tasks such as physical measurement or detection, while the gateway or high power devices perform complex tasks. In a multitier network, clustering /portioning are applied to decrease power dissipation and spreading complex computation task to rich nodes. In IWSN, several types of heterogeneous applications have been used with different requirements. Therefore, it is important to design a prototype with flexibility, reliability and hierarchical network that can fulfill the industries requirements in the same infrastructure.

Another important point is data aggregation in IWSN, processing the raw information within the sensor and sends the final decision to a destination node. This process will improve the power consumption of sensor different standards for IWSN such as ZigBee [6], WirelessHART [7], ISA100.11a [8] based on IEEE802.15.4 standard. The IEEE 802.15.4 is designed for large networks, especially where the synchronization is needed. The physical and MAC or routing layers influence the contention of resources. 
The physical layer has a direct impact of multiple access on sensor nodes and their wireless channels with interference level of the receiver side. In addition, the MAC layer obtains the allocated bandwidth for each transmitter and direct relation with a physical layer in terms of signal detection. Therefore, much technical and quality of services requirements are needed in IWSN. The prototype of IWSN should have the abilities of scalability, flexibility, reliable to handle and deal with the harsh industrial environment.

\subsection{IEEE802.15.4}

The new revolution of raw data processing within nodes is avoiding physical problems of an environment. Also, cost reducing based on artificial intelligent technique utilized for diagnosis the fault within sensors. However, because the harsh environments the wireless sensor require high power for the retransceiver data due to path loss, bit error, and flooding. Moreover, big data is the main challenge of the WSN that is utilized in an industry such as vibration data. On another hand, nodes distribution, easy maintenance and scheme reliability should take in account. Various working groups have established different standards for IWSN such as ZigBee, WirelessHART, and ISA100.11a based on IEEE802.15.4 standard. The IEEE 802.15.4 is designed for large networks, especially where the synchronization is needed. The ZigBee has not been designed for reliable real-time cyclic communication. On the other hand, WirelessHART is first open standard specially designed for real-time applications in industries. The ISA100.11a adopts the complete IEEE 802.15.4 PHY layer, however at the MAC layer standard has emerged TDMA and channel hopping to control the access of network. The ISA 100.11a and WirelessHART have been used for condition monitoring and best in terms of latency requirements.
Willig [9] was considered the performance of standard IEEE802.11 for network control systems and observed that the data transmission is impossible if the channel of the sensor is outside the scope of work. Likewise, this standard (802.11) is used for small data exchange between sensors of the wireless network. O'Rourke and Brennan [10], interested in packet optimization of standard 802.15.4 to enhance WSN communications performance. In another research [11] author setup a number of IEEE 802.11.4 nodes, which are capable to receive the information from the one source in the wireless network, whenever a copy of packet from other nodes (receivers) is requested, if the data packet do not decode from one of the nodes (receivers). In short message or not big data the IEEE 802.11.4 standard is more convenient for IWSN.

\subsection{Challenges for WSN in Industries}

Different types of WSN based applications have been designed for industries. These applications have various advantages over traditional wired systems. However, the industrial WSN based applications have strict requirements for data delivery and reliable communication. Due to harsh environment of industries, these types of usages have been challenged wireless communications problems such as path loss, delay time proceeding, packet loss and data flood[12]. These problems effected the reliability of data. Realizing excessive reliability is a major aim for applications in industries plants, where signals are disturbed from signal shadowing and multi-path fading because human and machinery effected. Another challenge is data collision in industries, due to unpredictability of wireless channel[13]. Furthermore, the diverse range of applications and their different requirements in industries, there is a need to improve reliability, delay in data delivery. 
However, to overcome these challenges are not straightforward due to the harsh industrial environment, strict budget, limited computational capabilities associated with sensor nodes. Routing decisions are a significant factor to improve data delivery, end-to-end delay and in time messages for applications.

\subsection{RSSI of the Sensor Application}

Received signal strength indicator (RSSI)[14] the method is used to measure the received signal strength and indicate the sensor transmitted signals. This technique measures the power of a signal by transmitted power and also calculates the propagation loss. This method has been used for radio frequency signals for indoor localization in industries. The RSSI do not require any additional hardware and has low overhead capabilities. However, it has some issue of multipath fading, irregular signal propagation, and interference. For IWSN, the RSSI needs more considerable efforts to obtain an empirical radio propagation model.

\section{Industrial Wireless Sensors}

The sensors in IWSN have been used for sensing, data collection, wireless communication, fault diagnosis. The different kinds of wireless sensors are implemented with the generating power system as a chip. Mostly, the industrial wireless sensors have four items: microprocessor, transceiver, power battery, and physical sensor. The sensor is a hardware device for sensing the measurable response of physical affective such as vibration, flow, load, speed, and humidity. The sensor produces analog signals and converted into digital signals through converters and send to the processor for processing. There are various different sources in sensors consume power from a battery such as during signal conversion, signal conditioning. The processing unit points to the tiny storage space unit for process signal, monitoring and control for effected other components. The transceiver unit links the node with the WSN by radios. The IWSN sensors operate with different modes such as transmit, receive, idle and sleep. The power of the wireless sensor was limited based on the type of a battery and consider as a most important thing in industrial wireless networks. Seems, in IWSN the power consumption is categorized into three basic domains: sensing, processing and communication [15]. In addition, for IWSN multi-hop environment, every sensor node plays a role of router and originator. For this reason, the sensor node consumes more power. To address the power issues of the sensor, various studies have been proposed related to energy aware routing, hardware optimization.

The wireless vibration sensors were utilized in industrials environment for collection and analysis different physical data such as: machine acceleration, oil temperature, bearing vibration, etc. thus, these wireless sensors utilize to protecting and monitoring faults of industrial machine. Vibration fault detection and control via protection scheme becomes important for high precision machinery for stable operations and accuracies. The active vibration systems have various sensors to sense and react to vibration disturbances. The Table 1 shows some wireless vibration sensors mostly use in industries. The vibration measurement is the difficult task because it has modes and components like displacement, velocity, acceleration, and frequencies or time. These components measure in a different way, peak to average, peak-to-peak. 
Table 1: Some popular vibration sensor nodes for industries.

\begin{tabular}{|l|l|l|}
\hline $\begin{array}{l}\text { S/N } \\
\text { o }\end{array}$ & Sensor Name & Description \\
\hline 1 & CMSS & $\begin{array}{l}\text { Producted by SKF } \\
\text { company, which is used } \\
\text { with low-frequency } \\
\text { applications such as } \\
\text { vibration for bearing defect } \\
\text { detection. }\end{array}$ \\
\hline 2 & $\begin{array}{l}\text { MiniSense } \\
\text { 100 Vibration } \\
\text { Sensor }\end{array}$ & $\begin{array}{l}\text { Vibration sensor is used } \\
\text { with high sensitivity } \\
\text { machine and low frequency, } \\
\text { also it is low cost sensor. }\end{array}$ \\
\hline 3 & $\begin{array}{l}\text { ADIS16223 Digit } \\
\text { al Tri-Axial } \\
\text { Vibration Sensor }\end{array}$ & $\begin{array}{l}\text { This sensor uses for analog } \\
\text { devices to monitor vibration } \\
\text { in industries. }\end{array}$ \\
\hline 4 & $\begin{array}{l}\text { Parker Hannifin's } \\
\text { wireless vibration } \\
\text { sensors }\end{array}$ & $\begin{array}{l}\text { This type monitors vibration } \\
\text { and temperature fault of } \\
\text { machinery, and wirelessly } \\
\text { technique which is used to } \\
\text { design this sensor. }\end{array}$ \\
\hline 5 & MEMS sensor & $\begin{array}{l}\text { This sensor supported a } \\
\text { digital self-test mode, it } \\
\text { uses for vibration fault } \\
\text { detection. }\end{array}$ \\
\hline
\end{tabular}

\section{Real Time Data Collection in Industries}

Recently, in industries the WSN based monitoring systems have been used to monitor and control the machine devices. The industrial environment has unique characteristics, in order to acquire real-time data from machines is still a challenge. This challenge makes a demand to design an effective system to process the heterogeneous sensor signals, high reliability and fast data delivery[16]. Compared to wired based systems, WSN systems have limited resources in terms of limited radio bandwidth, battery power and computational capabilities. Therefore, to achieve these all requirements system has abilities to handle the industrial harsh environment. Several IWSN based applications have been designed for data acquisition and transmit the feature extraction to the main computer [17]. The feature extraction on sensor and fault diagnosis are effective alternate approaches for raw data transmission, these approaches are able to reduce the quantity of data, save sensor node energy. In most of the cases, it is not possible to obtain all the required information about fault diagnosis by a single sensor. For industries, this situation is more complicated because of machines noise and interference. Furthermore, the data fusion approach was proposed to improve measurement accuracy by combining the data from different sensors. This approach reduces the data amount and saves the processing power and energy.

The fault diagnosis system composed with different hardware components such as amplifiers, sensors, data acquisition board, processing computer. The system has some signal processing techniques such as Fourier transform (FFT), Low Pass Filter (LPF), Band Pass Filter (BPF) and kurtosis for data analysis[18]. The FFT is an algorithm for convert signals from its original domain. The LPF refers to a filter for passing the signals with a frequency lower than a certain cut-off frequency. The BPF refers to an electronic circuit allows signals between two specific frequencies to pass and discriminates against signals for other frequencies.

\subsection{Neural network}

The neural network refers to network of artificial neurons used for data processing. Basically, the neural network is consisting a large number of simple and high interconnected elements in architecture. The neural network has the capability to recognize patterns especially when the information is noisy or incomplete[19]. The neural network is designed for classifying input predefined classes and converts into more categorized groups matching to groups' similarity. The significant feature of the neural network is ability to representation procedures and systems real behaviors. The input data utilized to train the neural network to get the expecting output for generating 
map which is represented the learning system model. These networks are able to represent in real time manner to the changing state of a model and provide continues sensor input. The neural network is used for complete systems, where various sensors are implemented and have different types of possible faults in real time manner [20].

For analysis purpose, the neural networks are used in system classification. In addition, the healthy and faulty that is related to machine situation should classify for training the neural network based on these cases. The input is in compressed version or spectrum and out is a training map. The network is able to identify the arbitrary patterns among the set of possible states. The main objective of clustering is to distribute the set of patterns in classes based on similar statistical and geometrical properties. After the classes, the characteristics feature are identified and classified for new patterns. The main issue in clustering is an estimation of a number of classes. It selects the wrong number of classes imposes in the artificial structure on the data rather than elaborate its true characteristics. For vibration analysis, clustering is a more efficient idea when the state of operations and system protection.

\subsection{Fuzzy System}

The fuzzy systems refer to the control system, where a mathematical system analyses analog input values into logical variables. Fuzzy system is widely used in machines for making decision or controlling. In many cases, the neural networks and genetic approaches have been used with fuzzy logic. The fuzzy system was proposed in 1965 by Lotfi A.Zadah in California [21]. The fuzzy system processing the data based on the design steps of; input membership, design rule, select Fuzzy Inference System, and build the output Membership.

\subsection{Related Work}

Several types of signal processing, neural network, fuzzy design approached have been designed for diagnosis machines faults. In this section, we reviewed some approaches in detail.

Wang and Jianu [22] was proposed a sensing unit for vibration measurement and machinery monitoring. The system is based on microprocessors and able to detect faults in shafts and bearings. The proposed system adopted a digital encoder for recognized the shaft rotation speed. The digital encoder is based on correlation spectrum to automatically recognize the shaft speed. This system based on wavelet energy spectrum, which is utilized for detection vibration fault on bearing. The system first demodulated the bearing resonance signature through wavelet analysis and able to enhance feature characteristics and come with bearing faults. The author claimed that system has robust results due to spectrum method especially for non-stationary feature extraction. Figure (2) shows the proposed system block diagram.

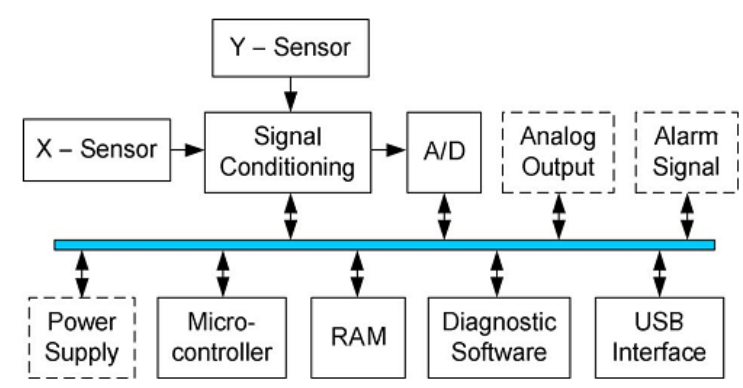

Fig.(2): Block diagram of sensing unit for vibration measurement[22].

Hou and Bergmann [23], developed IWSN for diagnosis and analysis the fault within sensor based on artificial intelligent techniques. The vibration signal and current used to process and analysis the fault of the induction motor. The neural networks were utilized for fault diagnosis and feature 
extraction. Moreover, the Dempster-Shafer (DS) scheme hybrid with two-stage classifier discovered to improve vibration fault detection precision. This new scheme demonstrated that the fault diagnosis vibration signal decrease $99 \%$ the payload broadcast and reduce $97 \%$ power consumption and enhance the real time processing.

In another induction motor fault diagnosis system was presented in [24], in which machinery fault diagnosis based on sensors feature extraction presented. Three types of motor operating conditions, mass imbalance, loose feet to evaluate the proposed system. The results show that on-sensor feature extraction and fault diagnosis effectively decrease payload transmission data, and node energy consumption. The system architecture uses star topology with one sink and two end nodes. The first node measures the $\mathrm{x}$ direction vibration and extracts the fault features and the second one extract the ydirection vibration. The system is based on ZigBee protocols. Figure (3) shows the block diagram of proposed system.

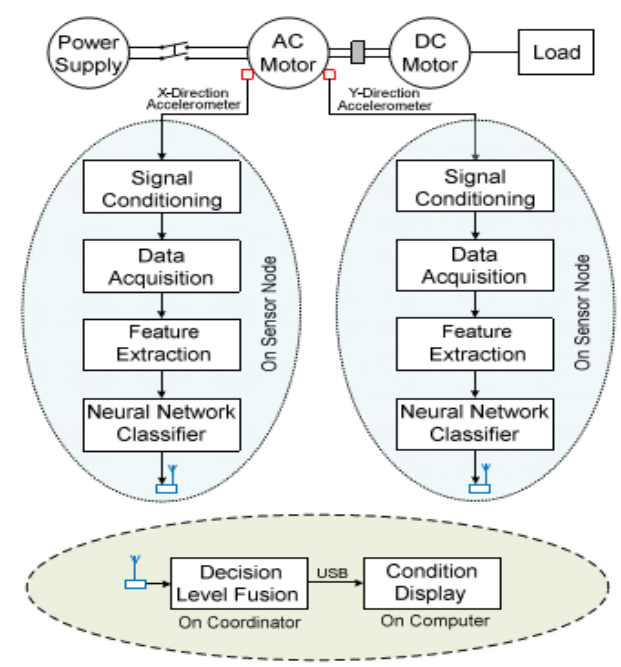

Fig.(3): Block diagram of wireless fault detection system [24].
Another method was proposed in [25], based on digital signal processor (DSP) wide range vibration detection and control. The main objective of proposed method is to suppress wide range vibration interruption that is related to signal in range sub-micron. The system implemented depend on utilized two wireless accelerometer sensors to diagnosis the vibration signal and feed these data to the controller. Furthermore, these sensors also responsible for attaining a wide range of detection at low cost. The results of this method showed that the method can achieve greater improvement compared to single sensor based systems. The single sensor based system has limited vibration range and resolution and cannot be able to achieve wide range vibration up to sub-micron level nanometre level. This method is only for active vibration application where the cheaper solution is needed.

Zhang, et al.[26] was presented a hybrid model for fault detection and bearing classification. The model is based on permutation entropy signal of vibration for detection the bearing faults. If the bearing fault is detected, the vibration signals are decomposed into a set of IMFs (intrinsic mode functions) through ensemble empirical mode decomposition. The PE values are calculated to attain multi-scale intrinsic features of vibration signals. The simulation results showed that the proposed method is effective and take multifarious fault types. In addition, the fault severity and load present that proposed method identified the level of severity and results are more accurate. The results also illustrated that proposed method is adaptable for real-time fault diagnosis applications.

Park, et al. [27] approached wireless vibration system for measurement vibration based on model consisted of wireless transceiver and electronic hardware. A 
portable data acquisition system is utilized to receive the information from sensors. The system can receive or send signal after amplified by control circuits and data acquisition system. The system feature is low-cost for monitoring the vibration data and decreases manpower for data acquisition. This system is a better to alternate to replace expensive systems. The results show the better performance of the system and provide accuracy of vibration data. However, due to the harsh environment of industries the Bluetooth communication is not suitable and has limitations.

Lei, et al. [28] was proposed another intelligent fault diagnosis system based on statistics analysis, adaptive neuro-fuzzy interference system (ANFIS) and distance evaluation approach. The system has three stages: different features improved distance evaluation and ANFIS to identify different abnormal cases. The different features including time statistical features, frequency, and empirical mode decomposition energy entropies are extracted to obtain fault information. The improved distance evaluation approach is used for most superior features selected from original features. The ANFIS identified the abnormal cases. The approach results show that proposed approach is reliable for recognize different faults in rolling bearings.

A classification system based on artificial intelligence approach and vibration signals for fault diagnosis is presented in [29]. The system has many neuro-fuzzy systems as a bi-classifiers with other equipment for vibration spectral data. In this proposed system, the faulty bearing detection system acquires the vibration signal produced by normal or faulty bearings. The data acquisition carries out with shaft rotation and collects the vibration information. Basically, the demodulated vibration signal along adaptive neuro-fuzzy system has used. The neuro-fuzzy has capable of selecting learning properties and expresses the resultant detection by rules. The results showed that the proposed system is better in the presence of several neuro-fuzzy systems in a biclassifier configuration and able to solve classification problem.

Weddell, et al. [30] was proposed a vibrationpowered sensing system for condition monitoring of machines. This system addresses the energy issues of wireless sensor nodes by proposed a vibration energy system accommodate variability in the power supply. The system provides a stable DC supply and support cold starting, overvoltage protection, adaptive operation and tracking the frequency changes of vibration source. The sensors in this system are used to monitor the vibration and temperature of the engine. In addition, the microcontroller provides an FFT on raw data, which is obtained from a digital accelerometer. The system has the ability to track dynamically the changings of vibration frequency. The system is tested on a ferry in order to verify the system ability.

Another effort for machine fault diagnosis established on wireless technique was suggested in [31]. In this system researcher classified his contribution into three stages: constructed new test bed, development wireless sensor and classification of vibration fault in bearings. In test bed, the vibration produced from bearings with unique frequency to limit the bearing condition. The wireless vibration sensors are utilized to collect the vibration signal from test bed. The wireless sensor is depended on IEEE 802.15.4 protocol standard. The analysis of vibration data obtains by two phases neural network are: generalized Hebbian and supervised vector quantization for the selforganizing map. The Figure (4) illustrations the wireless sensor module align with machine for wirelessly collecting the 
vibration signal to diagnosis the vibration fault.

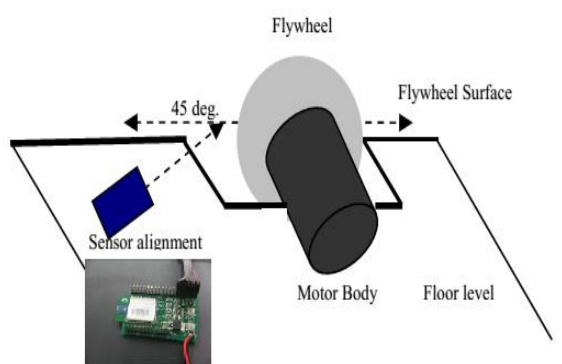

Fig.(4): Alignment of sensor with motor [31].

Awawdeh, et al. [32] investigated the application of WSN with accelerometer sensors for monitoring flow rate and patterns in large pipeline networks. In this application, the sensor nodes are tracking flow-induced vibration and estimate directly the flow change and provide means for fault detection. The experimental testbed composed with Tmote Sky sensors based on IEEE 802.15.4 protocol standard. The flowing fluid in pipelines causes of vibration and can be determined by analytically or numerically. Through this proposed system, the vibration and rotation flow rate analyze through deviation signals. The experiment results show that the system measures sensitivity and accuracy of flow rates and extract the features in order to improve monitoring and more comprehensive measurement of the pipeline system.

A vibration monitoring based on neural technology was designed in [33]. The system provides the approach to monitoring rolling element of bearings based on neural network technology. To process the data in neural networks the input data must be scaled. For design this system, the values are normalized into maximum and minimum values and computed scaled range. This system is composed with three modules: pre-processor the data compresses the data and performs actual classification of the signatures. The pre-processing is for identifying possibly faulty components. The low and highfrequency ranges in the proposed system provide more accurate detection of faults. In addition, the information provides to reduce training time by a factor of 10 . The system provides an efficient methodology in order to improve the vibration analysis and useful for monitoring and fault diagnosis in vibrations machines.

A stand-alone multi-sensor wireless system was proposed in [34] for continued monitoring of induction motors. The system provides a low-cost solution by dedicated current signature analysis or vibration. The system adopts multiple sensors platform to identify bearings damage, air gap statically or dynamically. For data analysis, system use two methods: Hilbert-Huang Transform (HHT) and Fast Fourier Transform (FFT). The HHT has the ability to analyze no stationary signals and FFT provides information to frequency domains. The relevant features carry the fault data based on signal processing and sensor algorithms. After several experiments, the proposed system detects almost $99 \%$ accurate faulty conditions. The combination of vibration signal and key frequencies of FFT yield high accuracy. From the results, it was noticed that extracted features and bearing failure are functions of rotational frequency. Figure 5 shows the block diagram of the classification algorithm. 


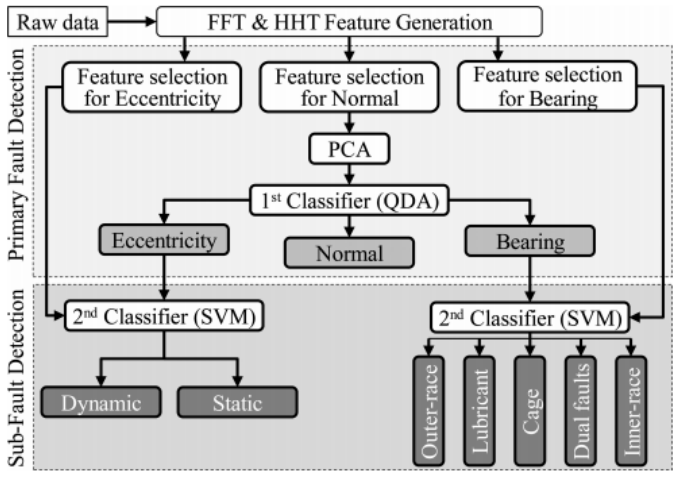

Fig.(5): Block diagram of classification algorithm [34].

Various different types of approaches have been proposed based on neural networks, fuzzy systems, FFT based, artificial intelligence. These systems have different limitations in terms of accuracy, processing, power consumption, etc.[4]. There is still a need to develop a system for the industrial harsh environment for fault diagnosis in order to improve the machines performance. The vibration-based condition monitoring approaches are the effective solution to maintain types of machinery and enhance industrial productivity. However, these approaches have been suffered from interference, large amount of data, obstacles issues in industries. The vibration signals always exhibit with non-stationary and nonlinear characteristics. These characteristics lead to difficulties to extract vibration signals. There is a need to design an effective system to deal with industrial harsh environments. Table 2 presents some more approaches with description.

Table 2: Machinery Fault Diagnosis Approaches.

\begin{tabular}{|c|c|c|c|}
\hline No & $\begin{array}{l}\text { Approach } \\
\text { Name }\end{array}$ & Description & Technique utilized \\
\hline 1 & $\begin{array}{l}\text { WSN based } \\
\text { Approach [35] }\end{array}$ & $\begin{array}{l}\text { WSN based approach was proposed for automation control } \\
\text { and monitoring based on embedded systems with ZigBee } \\
\text { technology. }\end{array}$ & Wireless technique \\
\hline 2 & $\begin{array}{l}\text { WSN based } \\
\text { vibration and } \\
\text { temperature } \\
\text { monitoring } \\
\text { approach [36] }\end{array}$ & $\begin{array}{l}\text { This approach was designed for a small wind turbine for } \\
\text { mechanical vibration and temperature monitoring. }\end{array}$ & Wireless technique \\
\hline 3 & $\begin{array}{l}\text { Fault detection } \\
\text { approach via } \\
\text { one-class v- } \\
\text { SVM vibration } \\
\text { [37] }\end{array}$ & $\begin{array}{l}\text { This method was designed to measure vibrational data } \\
\text { captured from windmill power machine based on one-class- } \\
\text { v-SVM method. }\end{array}$ & $\begin{array}{l}\text { Wireless and AI } \\
\text { technique }\end{array}$ \\
\hline 4 & $\begin{array}{l}\text { Distributed fault } \\
\text { detection system } \\
\text { for machine } \\
\text { monitoring [15] }\end{array}$ & $\begin{array}{l}\text { This approach was proposed for IWSN machines } \\
\text { conditioning monitoring based on intensive classifier in } \\
\text { computationally weak sensor network nodes. }\end{array}$ & $\begin{array}{l}\text { Wireless and unary } \\
\text { classification } \\
\text { technique }\end{array}$ \\
\hline 5 & $\begin{array}{l}\text { Hybrid } \\
\text { technique for }\end{array}$ & $\begin{array}{l}\text { The approach was designed for monitoring the machine in } \\
\text { terms of vibration fault diagnosis. In this approach, the raw }\end{array}$ & Wavelet technique \\
\hline
\end{tabular}


MJPS, VOL.(5), NO.(1), 2018

\begin{tabular}{|c|c|c|c|}
\hline & $\begin{array}{l}\text { machine } \\
\text { vibration and } \\
\text { monitoring } \\
{[38]}\end{array}$ & $\begin{array}{l}\text { signals are decomposed and FFT performed on vibration } \\
\text { signals. }\end{array}$ & \\
\hline 6 & $\begin{array}{l}\text { Fault diagnosis } \\
\text { scheme based } \\
\text { on multi-neural } \\
\text { network [39] }\end{array}$ & $\begin{array}{l}\text { This approach was proposed for fault diagnosis of turbine } \\
\text { vibration based on multi-neural networks. The different } \\
\text { diagnostic results are combined to obtain accuracy and } \\
\text { determine turbine failure. }\end{array}$ & Neural technique \\
\hline 7 & $\begin{array}{l}\text { Fault diagnosis } \\
\text { approach for } \\
\text { steam turbine } \\
{[40]}\end{array}$ & $\begin{array}{l}\text { This approach was proposed for steam turbine machines } \\
\text { based on particle swarm optimization and support vector } \\
\text { classifier approaches. In this approach, EPSO algorithm } \\
\text { automatically tunes the control parameters of an SVC. }\end{array}$ & SVC technique \\
\hline 8 & $\begin{array}{l}\text { Condition } \\
\text { diagnosis } \\
\text { approach }\end{array}$ & $\begin{array}{l}\text { This approach was designed for diagnosing the bearings } \\
\text { conditions based on intelligence algorithm hybrid from } \\
\text { statically method and back propagation scheme of NN. }\end{array}$ & Neural technique \\
\hline
\end{tabular}

\section{CONCLUSION}

In this review article, we have reviewed different approaches and systems for IWSN. Multiple wireless sensors are deployed on machinery in order to provide fault diagnosis and monitoring. Different systems have been designed to improve the industrial applications performance. The WSN are more applicable technologies for industrial automation monitoring and controlling the plant and equipment. However, the industrial requirement differs and complicated from other areas, due to its harsh environment. In recent year, various different types of efforts have been proposed based on particular applications. For fault diagnosis and vibration detection, various approaches with the fuzzy system, neural network, and signal processing were designed. We list and reviewed these approaches, industrial network types, sensors, standards, and challenges. Finally, we summarized these approaches in tabular form.

To design an industrial fault diagnosis system for industries from scratch is a tedious and time-consuming task. This review will help the researchers to pick the current situation in industries and analyze the proposed fault diagnosis approaches operations in industries.

\section{References}

[1] Liu, X., Ma, L. and Mathew, J.,2009, "Machinery fault diagnosis based on fuzzy measure and fuzzy integral data fusion techniques," Mech. Syst. Signal Process., 23, 3, 690-700,.

[2] Niu, G., Han, T., Yang, B. S. and Tan, A. C. C., 2007, Multi-agent decision fusion for motor fault diagnosis, Mech. Syst. Signal Process., 21, 3, 1285-1299.

[3] Yang, B. S. and Kim, K. J., 2006, Application of Dempster-Shafer theory in fault diagnosis of induction motors using vibration and current signals, Mech. Syst. Signal Process., 20, 2, 403-420.

[4] Gungor, V. C., Hancke, G. P. and Member, S., 2009, Industrial Wireless Sensor Networks: Challenges, Design Principles, and Technical Approaches, 4258 IEEE Trans. Ind. Electron., 56, 10, 4258-4265.

[5] Akyildiz, I., Melodia, T. and Chowdhury, K., 2007, A survey on wireless multimedia sensor networks, Comput. Networks, 51, 4, 921-960.

[6] Alliance, Z., 2006, "ZigBee Specification", ZigBee Document 053474r13. 
[7] Chen, D., Nixon, M. and Mok,A., 2010, why WirelessHARTTM, in WirelessHART, 195199.

[8] ISA100 Wireless Working Group, 2008, "Draft standard ISA100. 11a,".

[9] Willig, A.,2008, Recent and emerging topics in wireless industrial communications: A selection, IEEE Trans. Ind. Informatics, 4, 2, 102-122.

[10] Rourke, D. O' and Brennan, C., 2012, Practical packet combining for use with cooperative and non-cooperative ARQ schemes in resource-constrained wireless sensor networks, Ad Hoc Networks, 10, 3, 339-355.

[11] Ilyas, M. U., Kim M, Radha H, 2011, On enabling cooperative communication and diversity combination in IEEE 802.15. 4 wireless networks using off-the-shelf sensor motes. Wirel Networks 17:1173-1189.

[12]Akerberg, J., Gidlund, M. and Bjorkman, M., 2011, Future research challenges in wireless sensor and actuator networks targeting industrial automation, 2011 9th IEEE Int. Conf. Ind. Informatics, 410-415.

[13] Gharghan, S., Nordin, R. and Ismail, M., 2014, Energy-Efficient ZigBee-Based Wireless Sensor Network for Track Bicycle Performance Monitoring, Sensors, 14, 15573-15592.

[14] Zhang, X., Shrestha, R. and Wahid, K., 2015, An Efficient Algorithm for Localization Using RSSI Based on ZigBee, 366-369.

[15] Neuzil, J., Kreibich, O. and Smid, R., 2014, A Distributed Fault Detection System Based on IWSN for Machine Condition Monitoring, IEE Transaction on Industrial Informatics, 10, 2, 1118-1123.

[16] Hou, L. and Bergmann, N. W., 2010, System requirements for industrial wireless sensor networks, Emerg. Technol. Fact. Autom. (ETFA), IEEE Conf., 1-8.

[17] Salahshoor, K., Soleimani,M. and Kordestani, M., 2011, Fault detection and diagnosis of an industrial steam turbine using a distributed configuration of adaptive neuro-fuzzy inference systems, Simul. Model. Pract. Theory, 19, 5, 1280-1293.

[18] Hameed, Z., Hong, Y. S., Cho, Y. M., Ahn, S. H., and Song, C. K., 2009, Condition monitoring and fault detection of wind turbines and related algorithms: A review, Renew. Sustain. Energy Rev., 13, 1, 1-39, Jan.

[19] Schmidhuber, J., 2014, Deep Learning in Neural Networks: An Overview, 188, downloadfrom:

https://arxiv.org/abs/1404.7828 in May, 2016.

[20] Alianna, R. M. P,. Maren, J., Craig T Harston, 2014, Handbook of neural computing applications. Academic Press..

[21] Ikeda, H. and Hanamoto, T., 2013, Fuzzy controller of multi-inertia resonance system designed by Differential Evolution, Int. Conf. Electr. Mach. Syst., 2291-2295,

[22] Wang, W. and Jianu, O. A., 2010, A smart sensing unit for vibration measurement and monitoring, IEEE/ASME Trans. Mechatronics, 15, 1, 70-78.

[23] Hou, L. and Bergmann, N. W., 2012, Novel industrial wireless sensor networks for machine condition monitoring and fault diagnosis, IEEE Trans. Instrum. Meas., 61, 10, 2787-2798.

[24] Hou, L. and Bergmann, N. W.,2011, Induction Motor Fault Diagnosis Using Industrial Wireless Sensor Networks and Dempster-Shafer Classifier Fusion, in 2011 IEEE, 2992-2997.

[25] Li, H., 2015, A method of dual-sensor signal fusion for DSP-based wide-range vibration detection and control, Measurement, 69, 72-80.

[26] Zhang, X., Liang Zhou, Y., J. and Zang, Y., A novel bearing fault diagnosis model integrated permutation entropy, ensemble empirical mode decomposition and optimized SVM, Measurement, 69, 164179, 2015. 
[27] Park, C., Baek, R., Hoe, W., Kim, B. and Lee, H., 2014, Efficient Wireless Vibration Data Sensing and Signal Processing Technique Based on the Android Platform, 2014.

[28] Lei,Y., He,Z. and Zi, Y., 2008 , A new approach to intelligent fault diagnosis of rotating machinery, Expert Syst. Appl., 35, 1593-1600.

[29] Marichal, G., Artes, M., and GarciaPrada, J., 2011, An intelligent system for faulty-bearing detection based on vibration spectra, J. Vib. Control, 17, 6, 931-942.

[30] Weddell, A. S., Merrett, G. V., Barrow, S., and Al-Hashimi, B. M. ,2012, Vibrationpowered sensing system for engine condition monitoring, IET conference Publications, 1-5.

[31] Ballal, P., Ramani, A., Middleton, M., McMurrough, C., Athamneh, A., Lee, W., Kwan, C. and Lewis, F., 2009, Mechanical fault diagnosis using wireless sensor networks and a two-stage neural network classifier, IEEE Aerosp. Conf.

[32] Awawdeh, A., Bukkapatnam, S. T. S., Kumara, S. R. T., Bunting, C. and Komanduri,R., 2006 , Wireless sensing of flow-induced vibrations for pipeline integrity monitoring, IEEE Sens. Array Multichannel Signal Process. Work. Proceedings, SAM 2006, 114-117.

[33] Alguindigue,I. E., Loskiewicz-buczak, A. and Uhrig, R. E., 1993, Monitoring and Diagnosis of Rolling Element Bearings Using Artificial Neural Networks, IEEE Trans. Ind. Electron., 40, 2.

[34] Esfahani, E. T., Wang, S. and Sundararajan, V., 2014, Multisensor wireless system for eccentricity and bearing fault detection in induction motors, IEEE/ASME Trans. Mechatronics, 19, 3, 818-826.

[35] Sung, W. T., Hsu, Y. C. and Chen, J. H., 2011, Development of a Wireless Sensor Network for Factory Power Monitoring and Automation Control, Key Eng. Mater., 480481, 1346-1351.
[36] Spacek, a D., Junior, O. H. A., Neto, J. M., Coelho, V. L., Oliveira, Gruber, M. O., V. and Schaeffer, L., 2013, Management of Mechanical Vibration and Temperature in Small Wind Turbines Using Zigbee Wireless Network, IEEE Lat. Am. Trans., $11,1,512-517$.

[37] Martínez-Rego, D., Fontenla-Romero, O. and Alonso-Betanzos A., 2011, Power wind mill fault detection via one-class $v$ SVM vibration signal analysis, Proc. Int. Jt. Conf. Neural Networks, 10748, 511-518.

[38] Deng, L. and Zhao, R., 2013, A vibration analysis method based on hybrid techniques and its application to rotating machinery, Measurement, 46, 9, 3671-3682.

[39] Fei1, X., Hao, Z., and Daogang, P., 2014, Fault Diagnosis in Power Plant Based on Multi-Neural Network, IEEE Int. Conf. Syst. Sci. ang Eng.

[40] Sun, H.-C., Huang, C.-M. and Huang, Y.-C., 2013, Fault Diagnosis of Steam Turbine-Generator Sets Using an EPSOBased Support Vector Classifier, IEEE Trans. Energy Convers., 28, 1, 164-171.

[41] Wulandhari, L. a., Wibowo, A., and Desa, M. I., 2014, Condition diagnosis of multiple bearings using adaptive operator probabilities in genetic algorithms and back propagation neural networks, Neural Comput. Appl., 26, 57-65.

[42] Safizadeh, M. S. and Latifi, S. K., 2014, Using multi-sensor data fusion for vibration fault diagnosis of rolling element bearings by accelerometer and load cell, Inf. Fusion, $18,1-8$.

[43] Sun, H.-C., Huang, C.-M., and Huang, Y.-C., 2013, Fault Diagnosis of Steam Turbine-Generator Sets Using an EPSOBased Support Vector Classifier, Energy Conversion, IEEE Transactions on, 28, 164171.

[44] Wulandhari, L. A., Wibowo, A. and Desa, M. I., 2015, Condition diagnosis of multiple bearings using adaptive operator probabilities in genetic algorithms and back 
MJPS, VOL.(5), NO.(1), 2018

propagation neural networks, Neural Computing and Applications, 26, 57-65.

[45] Safizadeh, M. and Latifi, S., 2014, Using multi-sensor data fusion for vibration fault diagnosis of rolling element bearings by accelerometer and load cell, Information Fusion, 18, 1-8. 